\title{
Pérdida económica del ingreso familiar ocasionada por el gasto hormiga en las familias del fraccionamiento residencias de Mexicali, B. C.
}

\author{
Gloria Muñoz del Real ${ }^{1}$ \\ Alma Delia Inda ${ }^{2}$ \\ María del Socorro Barraza Pérez ${ }^{3}$
}

\begin{abstract}
Resumen
Esta investigación centra su interés en el estudio de la economía familiar, específicamente en los residentes del fraccionamiento Residencias del municipio de Mexicali Baja California, el estudio del ahorro y la generación de presupuestos en los gastos de las familias mexicalenses es considerado un punto de suma importancia para el control del ingreso familiar. La economía actual ha limitado el tiempo dedicado por los jefes de familia a las labores como lo es la preparación de alimentos en casa, lo que provoca recurrir a la adquisición de alimentos preparados. Esta problemática nos conlleva a preguntar si ¿la economía familiar se ve disminuida por el gasto hormiga en el consumo de alimentos preparados fuera de casa? La respuesta se orienta a afirmar que el impacto en el deterioro del ingreso familiar si se ve disminuido por el gasto hormiga en la compra de alimentos preparados, un resultado significativo en este sentido fue que el $57.5 \%$ de los residentes encuestados gasta más de lo contemplado en comida rápida, lo cual desequilibra el ingreso familiar.
\end{abstract}

\begin{abstract}
This research focuses its interest in the study of the family economy, specifically residents fractionation residences municipality of Mexicali Baja California, the study of saving and generating budgets in the costs of Mexicali families is considered a very important point for control of household income. The current economy has limited the time spent by householders to work, as is the preparation of food at home, causing recourse to the acquisition of prepared foods. This problem leads us to ask what if consumer spending ant food prepared outside the home diminishes the family economy? The answer is directed to say that the impact on the deterioration of family income if the ant spending on buying diminishes it prepared foods; a significant result in this regard was that $57.5 \%$ of residents surveyed spend more than contemplated fast food, which unbalances the family income.
\end{abstract}

Keywords: Economy, Ant Expenses Family Income and Savings.

Palabras clave: Economía, Gastos Hormiga, Ingreso Familiar y Ahorro.

\footnotetext{
${ }^{1}$ Contador Publico. Licenciada en Administración de Empresas. Licenciada en Mercadotecnia. Académico de la Universidad Autónoma de Baja California, gloria.munoz@uabc.edu.mx.

${ }^{2}$ Contador Publico. Coordinadora de Idiomas Extranjero. Académico de la Universidad Autónoma de Baja California, alma.inda@uabc.edu.mx.

${ }^{3}$ Contador Publico. Maestria en Contaduría. Académico de la Universidad Autónoma de Baja California, cpsocorrobarraza@uabc.edu.mx
} 


\section{Introducción}

La economía familiar y su administración adecuada es de suma importancia, en el presente trabajo se determina la consecuencia de los gastos innecesarios (gasto hormiga), en los que los residentes del fraccionamiento Residencias de Mexicali Baja California se ven perjudicados, el factor determinante en esta investigación fue el gasto en los alimentos preparados fuera de casa el cual constituye un derrame económico significativo. La problemática en cuestión no ha sido reconocida por completo por los residentes lo que nos lleva a analizar la pérdida económica del ingreso familiar, entonces se analizó y se planteó la siguiente pregunta ¿la economía familiar se ve disminuida por el gasto hormiga en el consumo de alimentos preparados fuera de casa?

Del Río (1997) Menciona que la economía no es sino el estudio de la humanidad en su conducta en la vida cotidiana (y de la sociedad en que vive). Es decir la economía es el estudio de la riqueza (y de la pobreza). ¿Quién, dónde, cómo y cuándo produce la riqueza, cómo se distribuye, quiénes la aprovechan y de quiénes se aprovechan? (¿y cómo le hacen?), ¿cuál es la relación? entre lo que producimos y lo que gastamos para comprar lo producido

Al realizar una investigación enfocada a los residentes antes mencionados se recaudó información donde se puede inferir que la mayoría de los residentes destina un gran porcentaje de su ingreso al gasto hormiga, donde se obtuvieron los siguientes resultados, el $57.5 \%$ de los residentes encuestados no presupuestan lo que gastan en comida rápida, lo cual desequilibra el ingreso familiar. Durante el estudio surgió un hallazgo importante el cual se refiere al presupuesto que realizan los residentes y sus gastos no presupuestados, causa de disminución en su economía.

\section{Desarrollo: revisión literaria}

Dado que el interés de esta investigación se centra en la economía y la cultura en el núcleo familiar para cuidar, será necesario plantear algunos parámetros que sirvan de ejes conceptuales sobre los que se apoya la lectura interpretativa del corpus. Para empezar, entenderemos el concepto de algunos autores que se consideran relevantes para esta investigación.

Economía, Paschoal (2002) "menciona que la economía es el estudio de la humanidad en las actividades normales de la vida; examina la acción individual y social en sus aspectos más estrechamente ligados a la obtención y uso de las condiciones materiales de bienestar. Así, por una parte, es un estudio de la riqueza, y por la otra y más importante es una parte del estudio del hombre".

Por otro lado Mankiw N. (1998) la define como "el estudio del modo en que la sociedad gestiona sus recursos escasos. En la mayoría de las sociedades, los recursos no son asignados por un único planificador central, si no por medio de las acciones conjuntas de millones de hogares y de empresa. Los economistas estudian, pues, el modo en que toman decisiones las personas: cuanto trabajan, que compran, cuanto ahorran y como invierten sus ahorros". Al mismo tiempo estipula que "también estudian el modo en que se interrelacionan. Por ejemplo, examinan la forma en que la multitud de compradores y vendedores de un bien determinan conjuntamente el precio al que se vende éste y la cantidad que se vende".

Por lo tanto explica que la economía es el estudio de como la sociedad administra sus recursos para el bienestar, así como, cuanto es el dinero que obtienen y en que lo distribuyen. Para la investigación actual se consideró más adecuada la teoría del siguiente autor 


\section{Reportes de investigación}

Del Río (1997) establece que "La economía no es sino el estudio de la humanidad en su conducta en la vida cotidiana (y de la sociedad en que vive). O sea, la economía es el estudio de la riqueza (y de la pobreza). ¿Quién, dónde, cómo y cuándo produce la riqueza, cómo se distribuye, quiénes la aprovechan y de quiénes se aprovechan? (¿y cómo le hacen?), ¿cuál es la relación? entre lo que producimos y lo que gastamos para comprar lo producido, para entender mejor lo anterior es necesario conocer el objetivo de la economía. Mientras que Malvin (1987) nos explica que "El objetivo de la Economía como ciencia es el estudio de las relaciones sociales de producción, esto es, el estudio de las relaciones que establecen los hombres en el proceso de producción, distribución, cambio y consumo de los bienes materiales que satisfacen las necesidades de la sociedad humana en las diferentes etapas o fases de su desarrollo histórico".

Tomando en cuenta la relación que tiene el ingreso familiar con el objetivo de la economía antes mencionado, se considera importante analizar la definición que nos proporciona Lionel Robbins, donde comenta lo siguiente: "Estudiar la satisfacción de las necesidades humanas mediante bienes que siendo escasos tienen usos alternativos entre los cuales hay que optar, es decir, se encarga de estudiar la manera en que la gente utiliza los recursos para alcanzar las condiciones materiales del bienestar, enfocándose en la escases y sosteniendo que es indispensable optimizar tales satisfactores para saciar las necesidades sociales".

Habiendo analizado las diferentes definiciones se considera que el objetivo de la economía esencial en esta investigación es mencionado por el autor Spencer M. H., (1993) "Ocuparse del comportamiento de las personas en particular cuando compran, venden, producen y consumen".

Una vez entendido que es economía es importante conocer la manera en que se obtiene el Ingreso familiar, por lo que Gollás M. (1983), (El Colegio de México) lo define como "El ingreso total familiar generalmente lo componen los ingresos que provienen del trabajo, del capital y de las transferencias (pensiones, donativos). Las preguntas que trata de responder la descomposición por origen del ingreso son: de la desigualdad total,
¿Cuánto es atribuible a la desigualdad que se observa en el ingreso que se recibe por trabajo? ¿Cuánto a la que se observa en el ingreso por capital y cuánto a la que se observa en el ingreso por transferencia?, mediante procedimientos de descomposición de índices se puede calcular la contribución de cada tipo de ingreso a la desigualdad total y la forma como ésta depende de la desigualdad en cada tipo de ingreso", para obtener una definición más clara.

Robles M. (1998) nos menciona que "En general, el ingreso del hogar se define como la suma de los ingresos de todos sus miembros y los que se captan de manera conjunta, tanto en efectivo como en especie. En este marco, los dos principales conceptos de ingreso familiar son el ingreso total del hogar y el ingreso disponible del hogar". Es decir se debe tener un conocimiento claro de lo que se puede y o no disponer sin embargo no en todas las familias se lleva un control adecuado.

De acuerdo con la definición anterior se deben administrar los ingresos familiares, entonces según el Instituto de prevención del delito de Gobierno del Estado de México (2014). Éste menciona que "Muchas familias tienen problemas con el manejo del dinero. En ocasiones, gastan más de lo que generan como ingreso, compran cosas innecesarias o simplemente no tienen suficiente dinero ahorrado, por lo que no pueden afrontar los imprevistos que le surgen ni aprovechar oportunidades en momentos particulares. Pero, el desarrollo de un presupuesto es, sin lugar a dudas, una solución a su problema. Un presupuesto familiar es una herramienta útil de planificación y control, la misma le permite identificar sus fuentes de ingresos, sus gastos principales y hacer una comparación entre éstos para reconocer fallas y tomar medidas correctivas a tiempo".

${ }^{7}$ Ecured, conocimiento con todos y para todos, definición clásica según Lionel Robbins http://www.ecured.cu/Econom\%C3\%ADa\#cite_ref-3, consultado el día 04 de Julio de 2016.

\section{4 / Trascender, Contabilidad y Gestión}


Por ejemplo el empezar a realizar algún tipo de ahorro, pero, ¿En qué consiste, el ahorro?; para la realización del ahorro es necesario hacer un presupuesto por lo que la Comisión Nacional para la protección y Defensa de los Usuarios de Servicios Financieros (CONDUSEF) define al presupuesto como "Anotar el dinero que se tiene y lo que se gasta en un periodo. Presupuestar permite saber con cuánto se cuenta y a qué se destina cada peso de los ingresos. Así como identificar si se gasta más de lo que se tiene. Un presupuesto es una herramienta útil para organizar los ingresos y gastos".

Entonces se entiende por ahorro familiar según la Procuraduría Federal del Consumidor (Profeco, 2014) como el "Guardar una parte del ingreso para enfrentar a futuro una emergencia o para cubrir una necesidad o satisfacer un deseo, así como hacer un consumo responsable del agua, energía eléctrica, papel, gas, etcétera, evitando su desperdicio".

Al final de cada situación todo con lleva a una disminución de efectivo, la cual se puede lograr al identificar nuestros gastos, reconocer los gastos hormiga al realizar nuestras compras sería una buena iniciativa, por lo tanto es importante conocer su definición.

Para León J. (2014) "El ahorro es la diferencia entre el ingreso disponible y el consumo efectuado por una persona, una empresa, etc. Cuando hay ahorro, los ingresos disponibles son mayores a los gastos y se presenta un superávit de dinero. Si los gastos fueran mayores a los ingresos, se presentaría un déficit".

El ahorrar ahora establece una reserva para el futuro, con el ahorro se puede prever la autosuficiencia económica para conformar un capital, que por pequeño, cubrirá compromisos futuros como son:

La educación de sus hijos.

- El poder tomar unas vacaciones y viajar.

- La posibilidad de comprar una casa.

- Para su jubilación.

- Solventar emergencias médicas o de otro tipo.

- Ayudará a mejorar su situación financiera, por ejemplo al pagar sus cuentas pendientes y mejorando su calificación crediticia.

- Así también su ahorro lo puede invertir en usted mismo, en su educación, entrenamiento laboral o para establecer algún tipo de negocio.
Y la última definición por parte de la Comisión Nacional para la protección y Defensa de los Usuarios de Servicios Financieros (CONDUSEF) nos dice que "Ahorrar es guardar una parte de los ingresos para utilizarla más adelante. Por lo tanto es importante destinar una parte del dinero al ahorro. El ahorro se puede usar, entre otras cosas, para pagar unas vacaciones, comprar una computadora, enfrentar una enfermedad o invertir en una vivienda. Es importante definir las metas que se quieren alcanzar, para así saber cuánto dinero se tiene que ahorrar y en cuánto tiempo se puede lograr".

Existen gastos que no se consideran al momento de presupuestar, los llamados gastos hormiga pero aun sí se realizaron, entonces por lógica se consideran como gastos innecesarios u hormiga. Según (Tenorio, 2012) Los gastos hormiga "son todos los gastos que no están reconocidos en el presupuesto, pero que si se realizan de manera cotidiana. El gasto hormiga representa desde lo máximo que serían artículos suntuarios (de lujo) hasta toma de ofertas en tarjetas de crédito (meses sin intereses), es decir, hormiga no quiere decir necesariamente pequeñitos sino también el gasto no necesario" y la Comisión Nacional para la Defensa de los Usuarios de las Instituciones Financieras define que "Los gastos hormiga son aquellos que se realizan día a día pero que no se les da importancia". No te ha pasado que llegas al final de la quincena y te preguntas ¿A dónde fue el dinero?, especialistas financieros aseguran que alrededor de $\$ 15,894.00$ (quince mil ochocientos noventa y cuatro pesos 00/100 m.n.) pesos anuales se pierden porque no administramos bien nuestro dinero.

Entre los gastos más populares están los cigarros, dulces, botanas, comidas fuera de la oficina, etc.

Por último la Profeco (2014) menciona que "cuando gastamos pequeñas cantidades de dinero en cosas como un paquete de chicles, o unas papas fritas, a primera vista nos puede parecer el gasto inofensivo, sin embargo, una vez sumados todos esos pequeños desembolsos la cuenta puede ser enorme y dañar nuestro ingreso seriamente; a esto nos referimos con gastos hormiga".

Núm. 2 / Mayo- Agosto del 2016/ 5 


\section{Reportes de investigación}

\section{Metodología}

Esta investigación se centra en el análisis del gasto hormiga ocasionado por los residentes del Fraccionamiento Residencias de Mexicali, Baja California, México, con el propósito de saber que tan consientes son las familias de este Fraccionamiento en cuanto a este tipo de gastos, ya que en su mayoría dichos residentes son empleados.

Para la realización de esta investigación se llevó a cabo la lectura de teorías, la elección de criterios para adecuarse correctamente a las categorías definidas por el marco teórico, la investigación fue de corte descriptivo y correlacional, misma que busca especificar y conocer propiedades y rasgos importantes del fenómeno que se analiza; el estudio a su vez plantea cuatro categorías que fueron medidas de manera independiente y poste- riormente descritas; el diseño de la investigación es de tipo transversal, debido a que se ha aplicado un instrumento (encuesta) en un momento determinado y en el lugar (Fraccionamiento Residencias donde viven los encuestados) pero sin involucrar el seguimiento de la investigación.

La encuesta que se utilizó en la investigación consta de 16 ítems de los cuales su respuesta son afirmaciones directas o específicas. Este instrumento puede contestarse de manera rápida y sencilla. Ver Cuadro No. 1. Construcción del Instrumento.

Cuadro 1: Construcción del Instrumento.

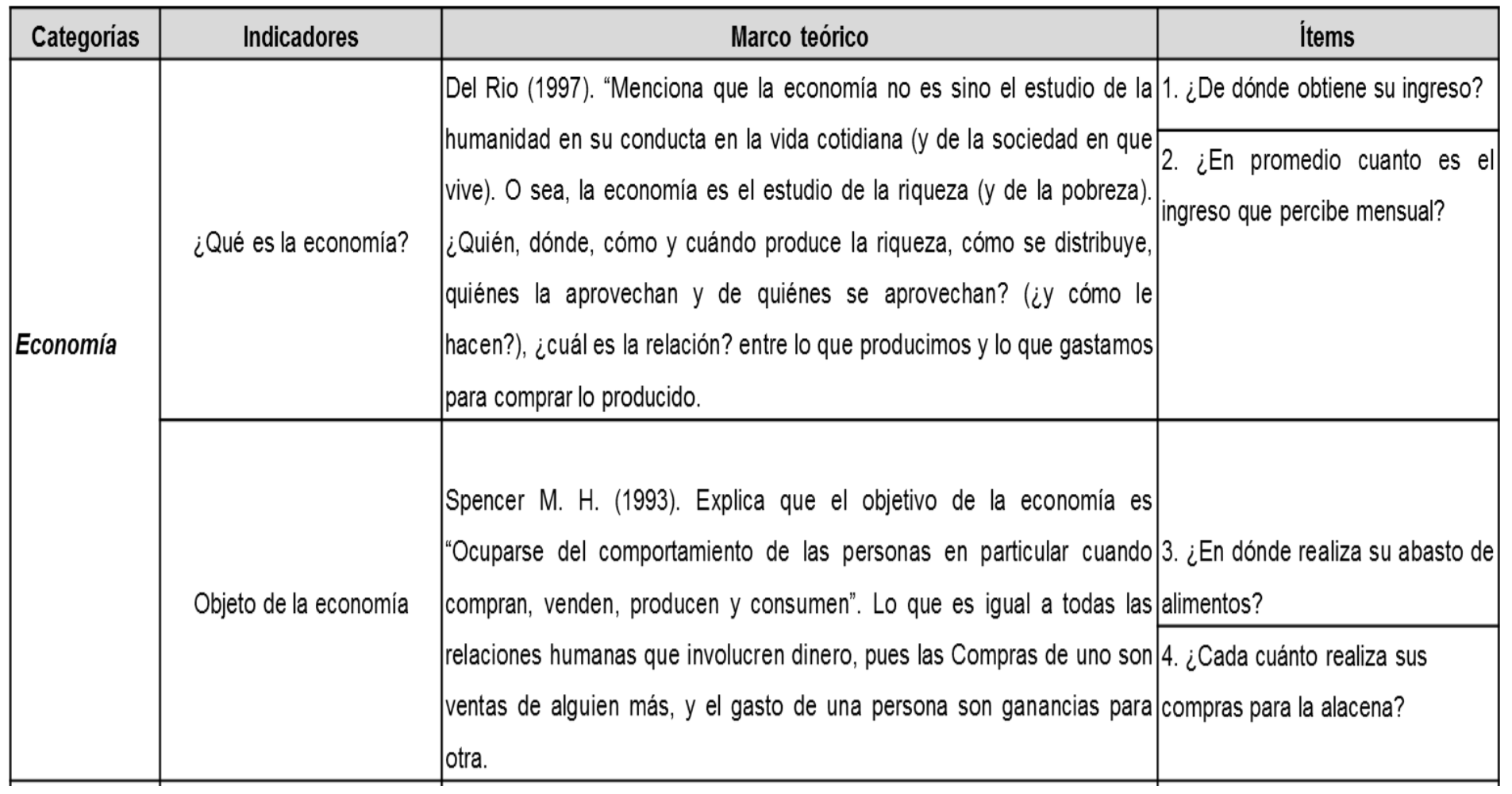

\section{6 / Trascender, Contabilidad y Gestión}




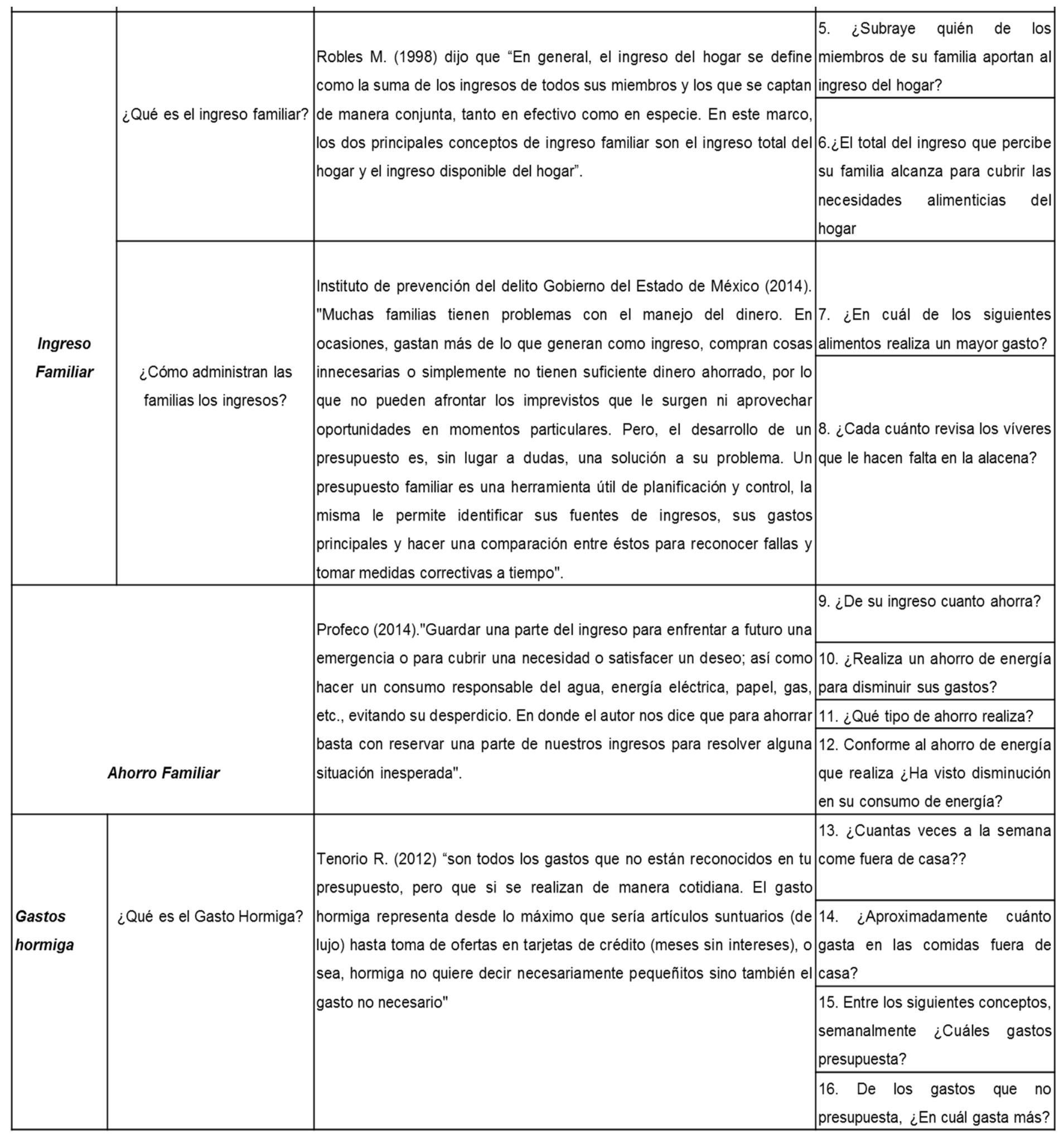

Fuente: Elaboración propia. 


\section{Reportes de investigación}

\section{Resultados}

Derivado del instrumento aplicado a la población sujeto de estudio, se encuentran los siguientes resultados:

Tabla 1: Estadística de obtención del ingreso.

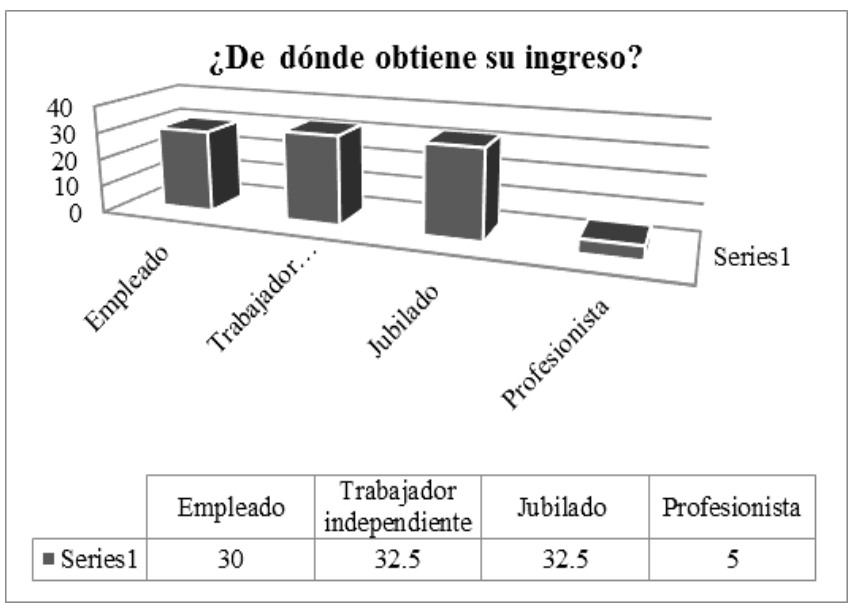

Fuente: Elaboración propia

Los habitantes del Fraccionamiento Residencias obtienen su ingreso de la siguiente manera; el $32.5 \%$ pertenece a jubilados y trabajadores independientes, el 30\% a empleados y un $5 \%$ a profesionistas.

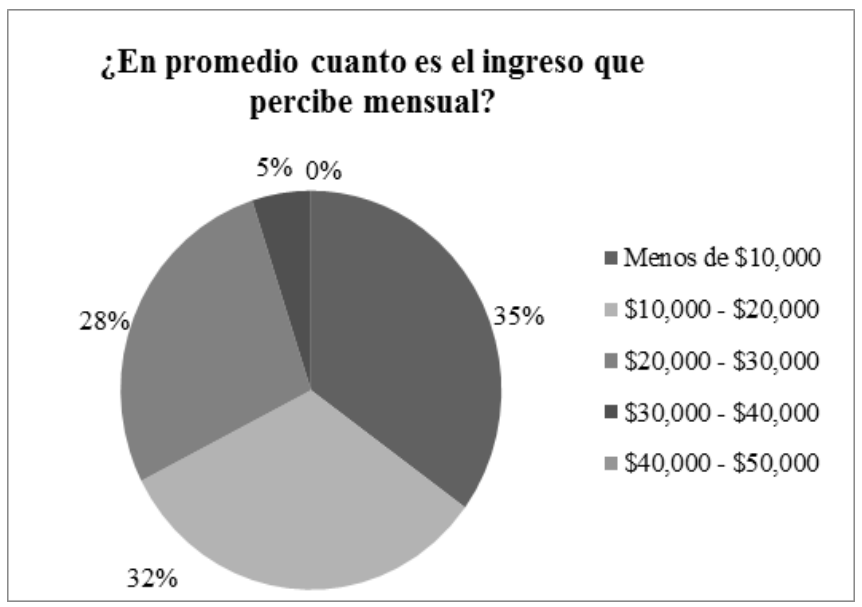

Fuente: Elaboración propia

De la muestra se obtuvo que el ingreso mensual en la mayoría es un $35 \%$ el cual pertenece al rubro de menor a 10,000 pesos, como segunda opción con un $32 \%$ el ingreso está entre 10,000 a 20,000 pesos, el $28 \%$ resultó que el ingreso es de 20,000 a 30,000 pesos y por último un $5 \%$ está entre 30,000 y 40,000 pesos mensuales.
Tabla 3: Lugar de abastecimiento de alimentos.

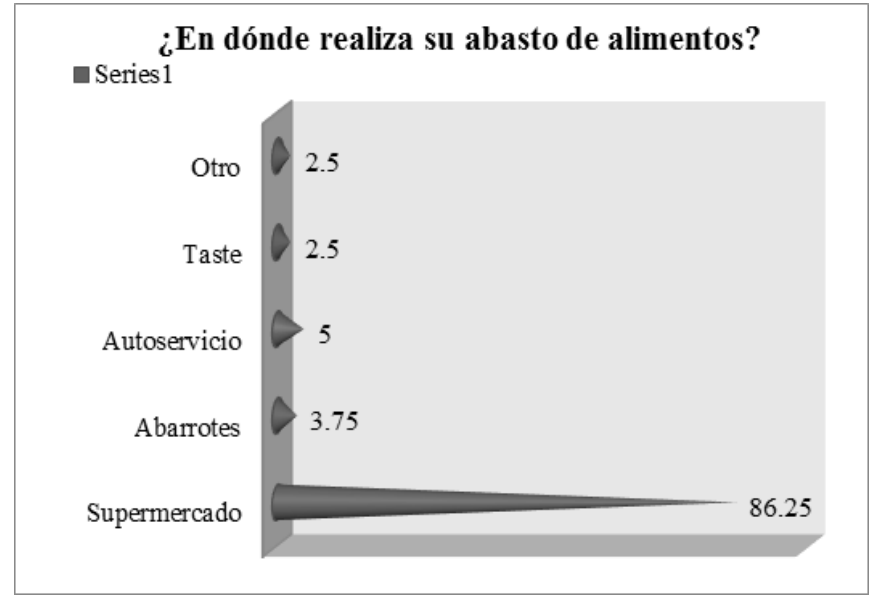

Fuente: Elaboración propia

La mayoría de los habitantes del Fraccionamiento Residencias abastecen sus alimentos en supermercados el cual tiene un porcentaje de $86.25 \%$, en un autoservicio esta el $5 \%$ de personas, en abarrotes un $3.75 \%$ y en Taste (Boutique de carnes) y otros lugares esta $2.5 \%$.

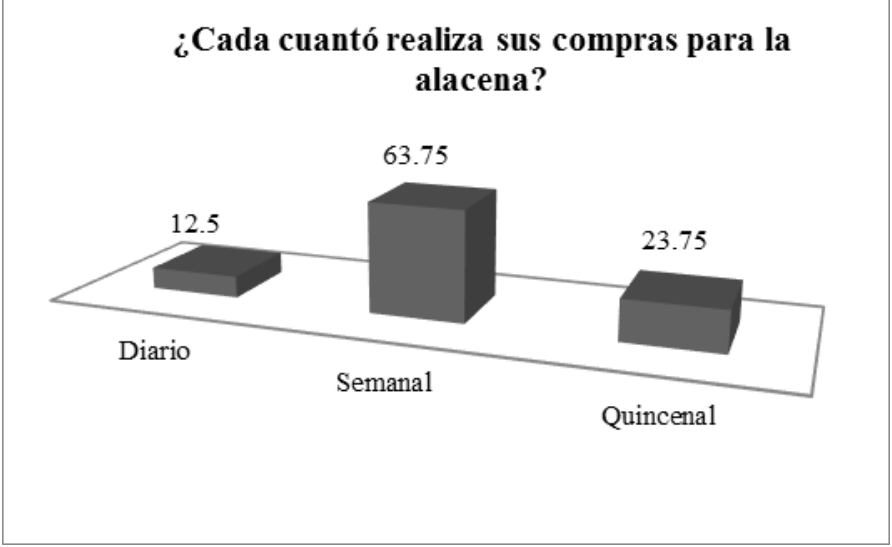

Fuente: Elaboración propia

Los habitantes del Fraccionamiento Residencias realizan sus compras semanales con un $63.75 \%$, la otra parte de los residentes compran de manera quincenal con un $23.75 \%$ y por último con un $12.5 \%$ lo hacen diario.

\section{8 / Trascender, Contabilidad y Gestión}


Tabla 5: Responsables del ingreso del hogar.

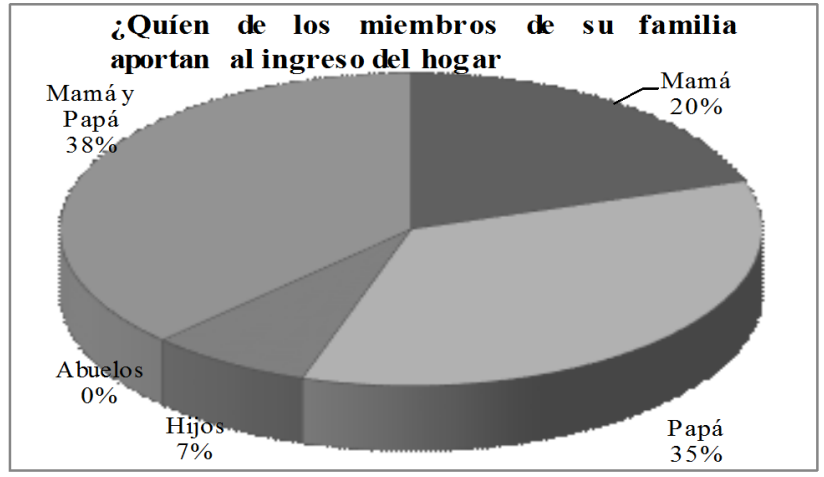

Fuente: Elaboración propia

En la mayoría de los hogares los que aportan el ingreso son los papás y las mamás con un $38 \%$, en otros hogares es el papá es el miembro que aporta al ingreso familiar con un $35 \%$, y por otra parte en ocasiones la mamá con

Tabla 6: Necesidades satisfechas por el total de ingresos.

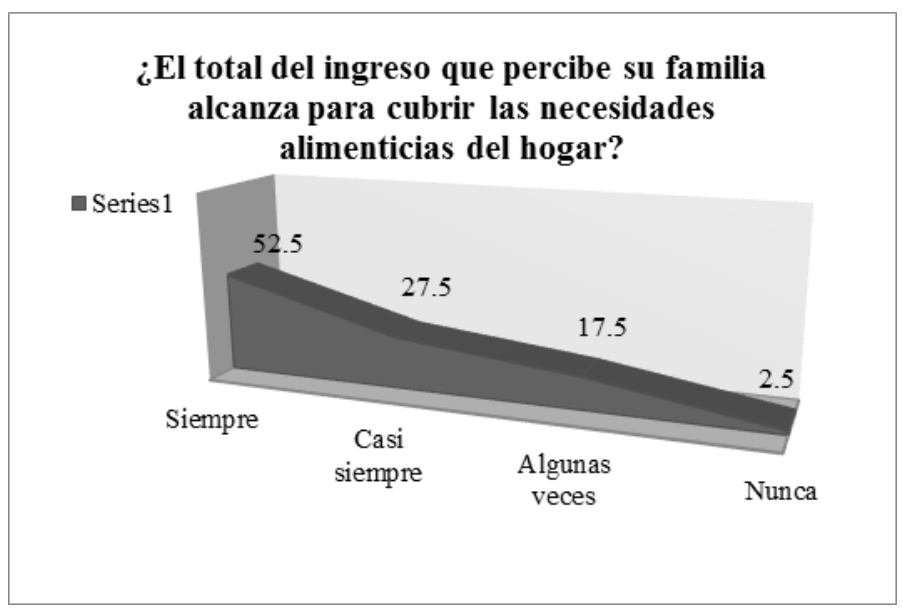

Fuente: Elaboración propia

Los residentes del Fraccionamiento Residencias informaron que el ingreso que perciben tan sólo a un $52.5 \%$ le alcanza siempre para cubrir sus necesidades alimenticias, un $27.5 \%$ dijo que casi siempre, el $17.5 \%$ algunas veces y el $2.5 \%$ nunca le alcanza para cubrir las necesidades alimenticias de su hogar.
Tabla 7: Estadísticas de alimentos que generan más gastos.

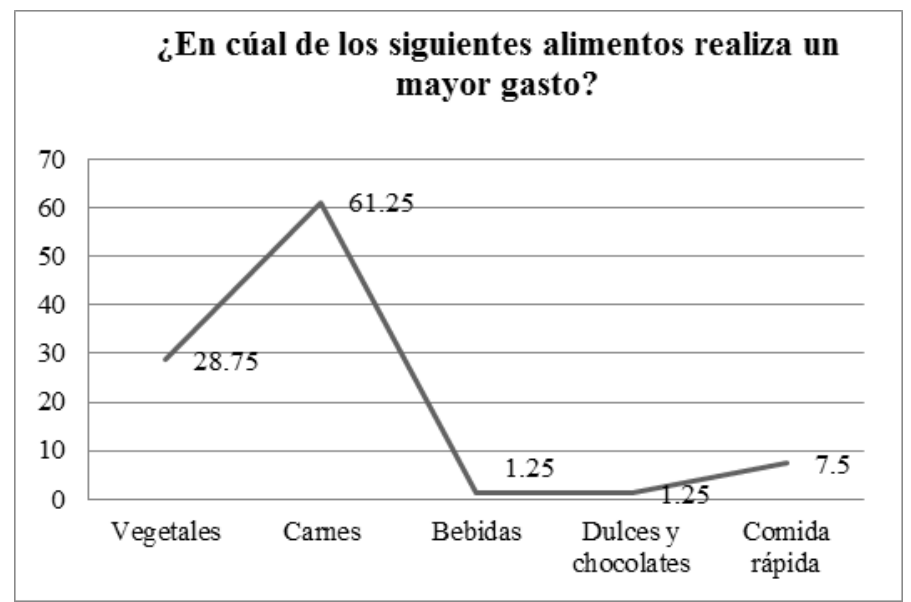

Fuente: Elaboración propia

La mayoría de los residentes realiza un mayor consumo en carnes con el $61.25 \%$ posteriormente están los vegetales con el $28.75 \%$, en comida rápida un $7.5 \%$ y por último están bebidas, dulces y chocolates con el $1.25 \%$.

Tabla 8: Tiempo de revisión de los víveres del hogar

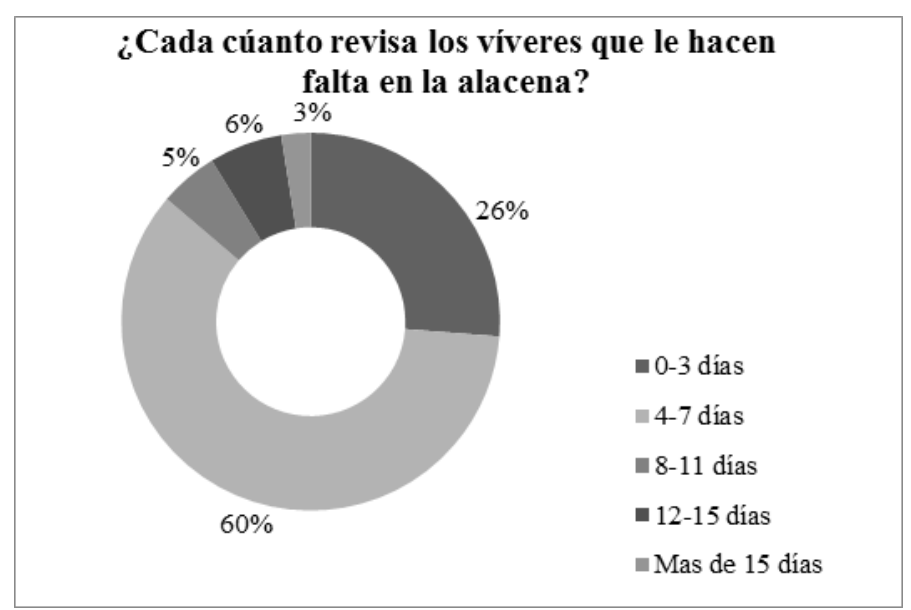

Fuente: Elaboración propia

El $60 \%$ de los residentes revisan su alacena de 4 a 7 días, el $26 \%$ lo realizan de 0 a 3 días, un $6 \%$ de 12 a 15 días, un $5 \%$ de 8 a 11 días y un $3 \%$ dentro de más de 15 días. Lo cual muestra la preocupación por mantener siempre los alimentos para los miembros de la familia. 


\section{Reportes de investigación}

Tabla 9: Estadística del ingreso ahorrado

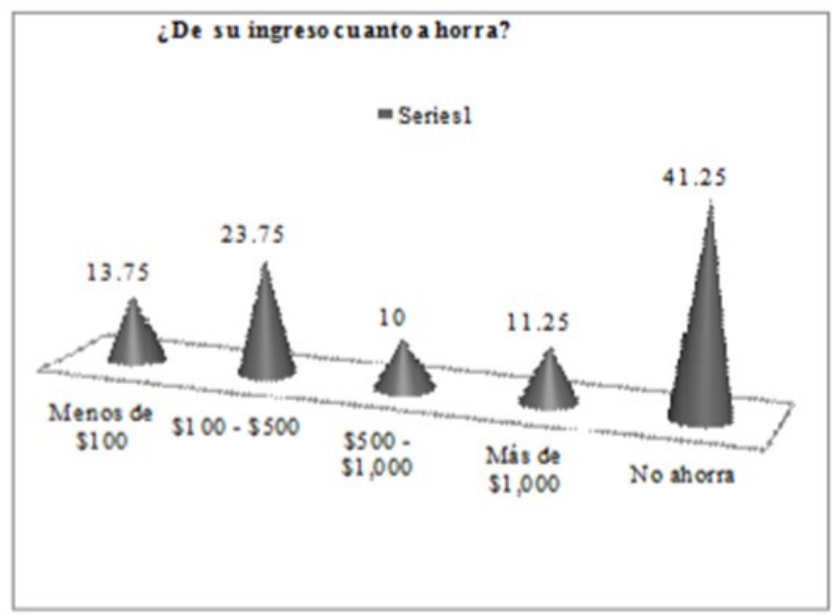

Fuente: Elaboración propia

El $41.25 \%$ de los residentes del Fraccionamiento Residencias no ahorra de su ingreso percibido, el $23.75 \%$ ahorra entre 100 a 500 pesos, un $13.75 \%$ ahorra menos de 100 pesos, un $11.25 \%$ ahorra más de 1000 pesos y con un $10 \%$ ahorran entre 500 a 1000 pesos. Esto muestra la poca cultura de ahorro en el ingreso que percibe la familia.

Tabla 10: Porcentaje de habitantes que ahorran energía

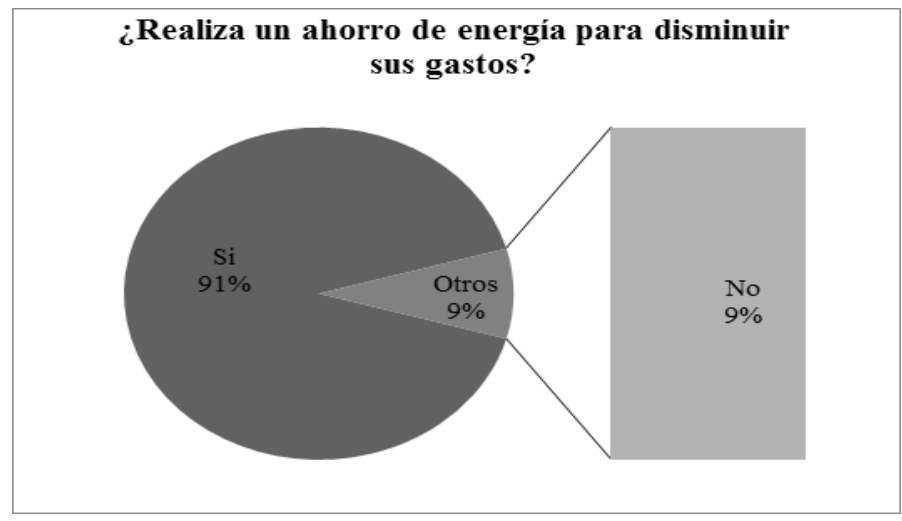

Fuente: Elaboración propia

En el Fraccionamiento Residencias el 91\% realiza un ahorro de energía para la disminución de sus gastos, el 9\% restante no lo hacen. Es decir, la mayoría de la población está preocupado por los gastos fijos que se generan en el hogar y trata de implementar alguna forma de consumo en los mismos.
Tabla 11: Tipo de ahorro de energía para disminuir gastos

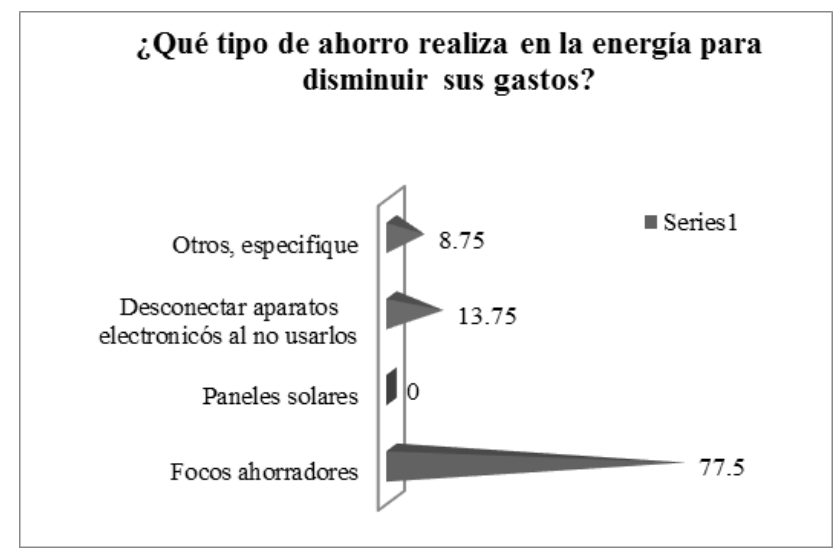

Fuente: Elaboración propia

EL $77.5 \%$ Usa focos ahorradores, el $13.75 \%$ desconecta aparatos electrónicos que no usen, y el $8.75 \%$ restante en otra forma de ahorro. Para los residentes es importante el ahorro de los gastos debido a que con ello incrementara la parte del segmento que separo para compra de víveres en el hogar y/o alguna contingencia que pudiese suscitarse.

Tabla 12: Disminución por ahorro de energía

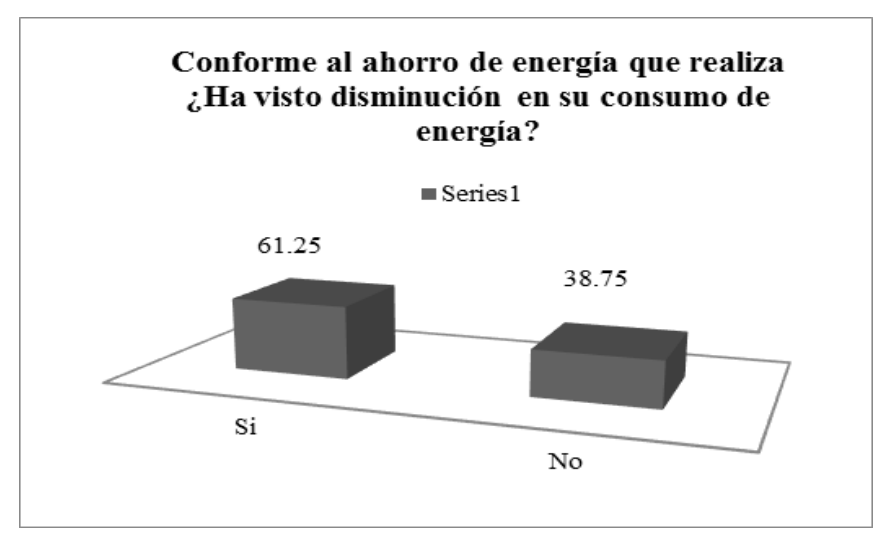

Fuente: Elaboración propia

La mayoría de los residentes que realizan un ahorro de energía contestaron que si han visto una diminución con un $61.25 \%$ lo restante dijeron que no con un $38.75 \%$. 
Tabla 13: Salidas a comer fuera de casa

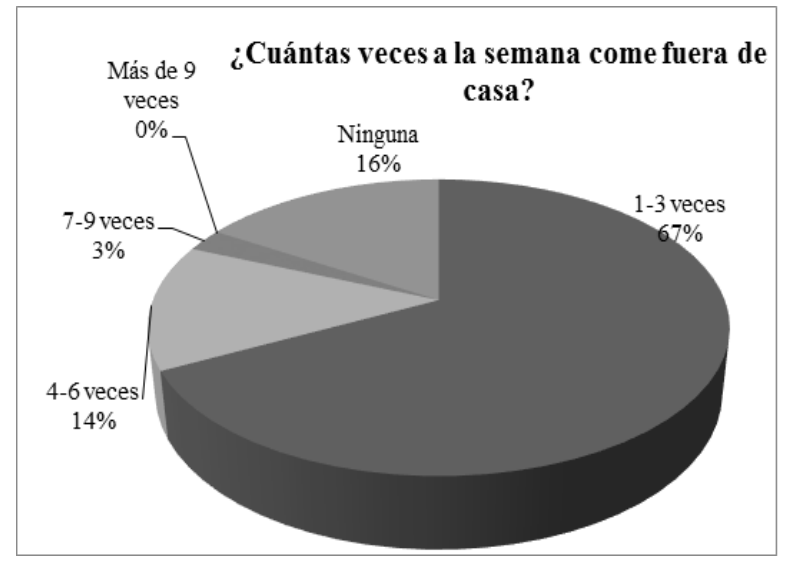

Fuente: Elaboración propia

De la muestra obtenida el $67 \%$ como fuera de casa de 1 a 3 veces a la semana, el 16\% ninguna vez, el $14 \%$ de 4 a 6 veces y el $3 \%$ restante de 7 a 9 veces a la semana. Esto quizá debido a la falta de tiempo que les permite su horario de trabajo o simplemente por lujo de algunos residentes del fraccionamiento.

Tabla 14: Gasto generado por comida fuera de casa

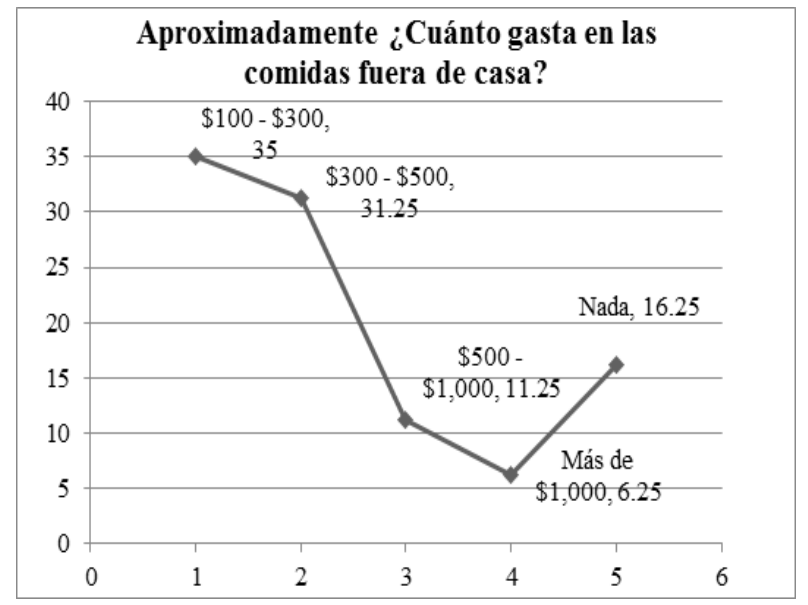

Fuente: Elaboración propia

El 35\% gasta en las comidas fueras de casa entre 100 y 300 pesos, el $31.25 \%$ entre 300 y 500 , el $16.25 \%$ no gasta nada, el $11.25 \%$ entre 500 y 1000 pesos y más de 1000 pesos el $6.25 \%$ de los residentes del Fraccionamiento Residencias.
Tabla 15: Gastos presupuestados

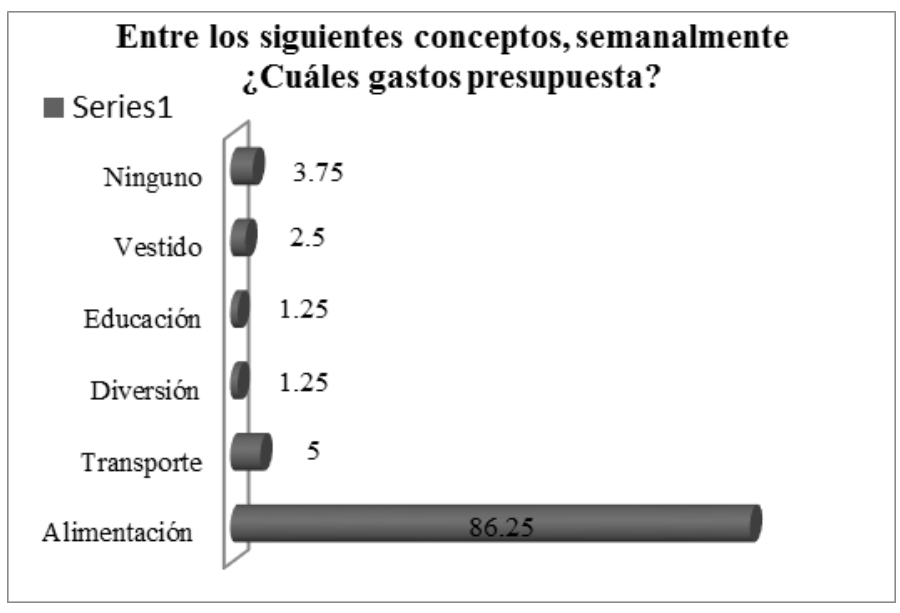

Fuente: Elaboración propia

La mayoría de los residentes con $86.25 \%$ presupuesta para la alimentación, un 5\% presupuesta para el transporte, un $3.75 \%$ no presupuesta nada, para la vestimenta un $2.5 \%$ para educación y diversión es $1.25 \%$. Prácticamente el gasto más significativo en el hogar es la alimentación, quizá por la importancia que este reviste.

Tabla 16: Gastos no presupuestados que generan más egreso.

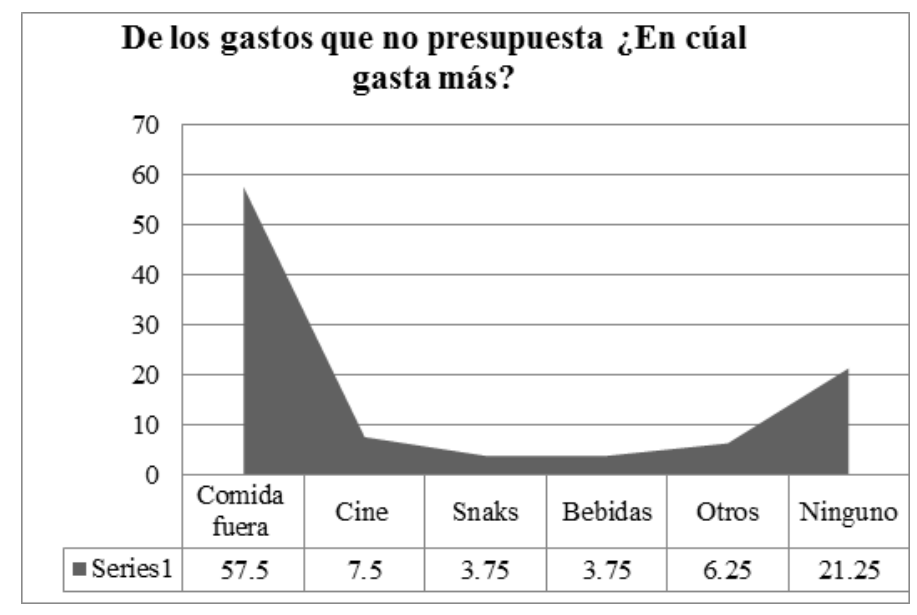

Fuente: Elaboración propia

El $57.5 \%$ gasta más en comida rápida, $21.25 \%$ ninguno, en el cine un $7.5 \%$ otros $6.25 \%$ y en snacks y bebidas el $3.75 \%$. 


\section{Reportes de investigación}

\section{Conclusiones}

La economía de las familias del Fraccionamiento Residencias de Mexicali, Baja California recae en los ingresos obtenidos en su mayoría por los padres de familia lo que significa que la conducta generalizada en el apartado de economía centra su interés en un ingreso unilateral, es decir, que los demás integrantes de las familias sólo son individuos que consumen de los ingresos de los padres y que no aportan a la manutención de su seno familiar.

Por otra parte también se encontró que los ingresos que perciben algunas de las familias del Fraccionamiento Residencias de Mexicali, B.C., son la suma de los ingresos de cada uno de los integrantes de la familia, las cuales tienen problemas con la administración de su ingreso ya que en ocasiones suelen gastar más de lo que ganan en cosas innecesarias.

Como resultado de la falta de administración de los ingresos que perciben las familias, realizan los llamados gastos hormiga y gracias a esta investigación se pudo encontrar que los residentes realizan gastos en exceso que no tienen contemplados en su presupuesto y de tal forma que esto ocasiona que a futuro no tengan suficiente dinero ahorrado para imprevistos.

Es necesario que las familias del Fraccionamiento Residencias, de Mexicali, Baja California, cuenten con un ahoro destinado para eventualidades que puedan suceder durante la semana. Deben presupuestar en las cosas que van a gastar y anotarlo o guardar las notas para llevar un control y no comprar cosas repetidas que no necesiten, o en su caso comprar cosas que sobrepasen su presupuesto.

Los residentes deben realizar algún plan de organización con los ingresos que perciben y así cuando quieran comer fuera de casa tengan destinado ya una cantidad de lo que se va a gastar, por otra parte también se les recomienda que organicen bien sus tiempos para que de esta manera sea menos frecuente las comidas fuera de casa.
Es de gran importancia que los residentes, realicen una buena organización dentro los días laborados, de escuela, etcétera. Para así poder evitar el gasto de alimentos preparados fuera de casa y tengan un mayor rendimiento de sus ingresos y de esta manera tener una buena salud.

\section{Bibliografía}

Condusef. (2012). Gasto Hormiga. 9 de marzo de 2015, de Comisión Nacional para la Defensa de los Usuarios de las Instituciones Financieras. Sitio web: http:// konexiondocente.mx/wp-content/uploads/2012/11/ hormiga.pdf

CONDUSEF. (2014). Guía Familiar de Educación Financiera. 10 de marzo de 2015, de Comisión Nacional para la protección y Defensa de los Usuarios de Servicios Financieros. Sitio web: http:// www.condusef.gob.mx/PDF-s/educacion financiera/ familiar/guia familiar.pdf

Del Río (1997), La economía al alcance de todos. Diccionario de términos económicos.

Ecured, conocimiento con todos y para todos, definición clásica según Lionel Robbins, Sitio web: http:// www.ecured.cu/Econom\%C3\%ADa\#cite ref-3, 04 de Julio de 2015.

Gollás M. (1983), La desigualdad del ingreso familiar en México: Origen y causas (El Colegio de México). Sitio Web: http://aleph.academica.mx/jspui/

bitstream/56789/6115/1/

DOCT2065327 ARTICULO 13.PDF

Instituto de prevención del delito. (2014). Como administrar los ingresos del hogar. 11 de Marzo de 2015, de Instituto de prevención del delito Sitio web: http:// portal2.edomex.gob.mx/idcprod/groups/public/ documents/edomex archivo/ ipd pdf ingresos archivo.pdf 
León J. (2014). El ahorro familiar. 12 de marzo de 2015, de Mutualista Pichincha Sitio web: http:// www.mutualistapichincha.com/mupi/Documentos/

AdministrandoHogar/AhorroFamiliar.pdf

Malvin, E.E. (1987). Economía para principiantes. México: CECSA.

Mankiw N., Gregory. (1998). Principios de economía. México: Mc Graw Hill.

Paschoal Rossetti José. (2002). Introducción a la economía. México: Alfaomega-Oxford.

Profeco. (2014). Brújula de compra. 9 de marzo de 2015, de Procuraduría federal del consumidor Sitio web: $\quad$ http://www.profeco.gob.mx/encuesta/brujula/ bruj 2014/bol290 gastos hormiga.asp

Profeco. (2014). Gastos hormiga. 05 de octubre de 2014, de Procuraduría federal del consumidor Sitio web: http://www.gob.mx/profeco/documentos/gastoshormiga?state $=$ published

Profeco. (2014). Guía de Educación Financiera para un Consumo Responsable. 12 de Marzo de 2015, de Procuraduría federal del consumidor Sitio web: http:// www.consumidor.gob.mx/wordpress/wp-content/ uploads/2014/11/Guia de Educacion Financiera.pdf

Robles M. (1998). Determinación del ingreso familiar. Encuesta Integrada de hogares 1997 - 1998. 10 de marzo de 2015, de Secretaría Técnica De Planificación Del Desarrollo Económico Y Social Sitio web: http:// www.cepal.org/deype/mecovi/docs/TALLER13/17.pdf
Spencer, M. H. (1993). Economía contemporánea (3a ed.).

Barcelona, España: Reverte. Sitio web: http:// roa.uveg.edu.mx/repositorio/licenciatura/182/

EconomavsEcologa.pdf

Tenorio R. (2012). Cuidado con el gasto "hormiga". 9 de Marzo de 2015, de Sergio Meana Sitio web: https:// intranet.ebc.edu.mx/contenido/vidaebc/archivos/ publicaciones/30jul12.pdf 


\section{Anexos}

Dirección Genero Edad y Escolaridad

1. ¿De dónde obtiene su ingreso?

\begin{tabular}{|l|l|l|l|}
\hline Empleado & Trabajador independiente & Jubilado & Profesionista \\
\hline
\end{tabular}

2. ¿En promedio cuanto es el ingreso que percibe mensual?

\begin{tabular}{|l|l|l|l|r|}
\hline Menos de $\$ 10,000$ & $\$ 10,000-\$ 20,000$ & $\$ 20,000-\$ 30,000$ & $\$ 30,000-\$ 40,000$ & $\$ 40,000-$ \\
$\$ 50,000$ & & & \\
\hline
\end{tabular}

3. ¿El total del ingreso que percibe su familia alcanza para cubrir las necesidades alimenticias del hogar?

\begin{tabular}{|l|l|l|l|}
\hline Siempre & Casi siempre & Algunas veces & Nunca \\
\hline
\end{tabular}

4. ¿En cuál de los siguientes alimentos realiza un mayor gasto?

\begin{tabular}{|l|l|l|l|l|}
\hline Vegetales & Cames & Bebidas & Dulces y chocolates & \\
Comida rápida
\end{tabular}

5. Subraye quién de los miembros de su familia aportan al ingreso del hogar.

\begin{tabular}{|l|l|l|l|l|}
\hline Mamá & Papá & Hijos & Abuelos & Mamá y papá \\
\hline
\end{tabular}

6. ¿Cada cuánto revisa los víveres que le hacen falta en la alacena?

\begin{tabular}{|l|l|l|l|l|}
\hline $0-3$ dias & $4-7$ días & $8-11$ días & $12-15$ días & Más de 15 dias \\
\hline
\end{tabular}

7. ¿En dónde realiza su abasto de alimentos?

\begin{tabular}{|l|l|l|l|l|}
\hline Supermercado & Abarrotes & Autoservicio & Taste & Otro \\
\hline
\end{tabular}

8. ¿Cada cuánto realiza sus compras para la alacena?

\begin{tabular}{|l|l|l|}
\hline Diario & Semanal & Quincenal \\
\hline
\end{tabular}

9. Entre los siguientes conceptos, semanalmente ¿cuáles gastos presupuesta?

\begin{tabular}{|l|l|l|l|l|l|}
\hline Alimentación & Transporte & Diversión & Educación & Vestido & Ninguno \\
\hline
\end{tabular}

10. De los gastos que no presupuesta ¿En cuál gasta

más?

\begin{tabular}{|l|l|l|l|l|l|}
\hline Comida fuera & Cine & Snacks & Bebidas & Otros & Ninguno \\
\hline
\end{tabular}

